\title{
Re-determination of Phoebe's orbit
}

\author{
K. X. Shen ${ }^{1,3}$, D. Harper ${ }^{2}$, R. C. Qiao ${ }^{1,3}$, G. Dourneau ${ }^{4}$, and J. R. Liu ${ }^{1,3}$ \\ ${ }^{1}$ National Time Service Center, The Chinese Academy of Science, PO Box 18, Lintong, Shaanxi 710600, PR China \\ e-mail: shenkx@ntsc.ac.cn \\ 2 Wellcome Trust Sanger Institute, Wellcome Trust Genome Campus, Hinxton, Cambridge CB10 1SA, UK \\ ${ }^{3}$ United Laboratory for Optical Astrometry, The Chinese Academy of Science, PR China \\ 4 Observatoire Aquitain des Sciences de l'Univers, UMR 5804 Laboratoire d'Astrodynamique, d'Astrophysique \\ et d'Aéronomie de Bordeaux (L3AB), 2 rue de l'Observatoire, 33270 Floirac, France
}

Received 20 January 2005 / Accepted 10 March 2005

\begin{abstract}
In order to improve the orbit of Phoebe, the ninth satellite of the Saturnian system, 101 new observations were made by our research team in 2003, using a CCD detector of large size $(2048 \times 2048$ pixels $)$ mounted on the $1.56 \mathrm{~m}$ astrometric reflector at the Sheshan Station of Shanghai Astronomical Observatory. We fitted a numerical integration of its orbit to all of the collected Earth-based astrometric observations from 1904 to 2003, including the newest precise data sets from Qiao \& Tang and from Peng et al. A new set of initial conditions of Phoebe has been obtained, leading to an improved orbit of this satellite.
\end{abstract}

Key words. gravitation - ephemerides - methods: numerical - reference systems - astrometry - time

\section{Introduction}

In comparison to the eight inner satellites of Saturn, the accuracy of the ephemeris of Phoebe, the ninth satellite, is poor due to the very small number of observations and their irregular distribution.

Recently, Qiao \& Tang (2004) developed a campaign for observing Phoebe at the Sheshan station (E 121.18417, N 31.09611) of the Shanghai Astronomical Observatory (SHAO) in 2003, using its $1.56 \mathrm{~m}$ astrometric reflector with a focal length of $15.6 \mathrm{~m}$, and they obtained a set of $101 \mathrm{new}$ CCD positions.

In this paper, we used this SHAO data and several other series of new CCD data published by Veiga et al. (2000), Fienga et al. (2002), Stone (2000, 2001), and Peng et al. (2004), together with observations from 1904 to 1993, to obtain a new determination of Phoebe's orbit.

\section{Method of reduction and perturbation}

At the beginning of the 20th century, the first preliminary orbital elements of Phoebe were given by Pickering (1905a,b). In the same year, Ross (1905) derived an analytical theory of the satellite's motion. Fifty years later, Zadunaisky (1954) made an attempt to update the orbit model of Ross using a larger set of observational data. Elmabsout (1970) developed a semianalytical theory. However, Ross's theory remains the basis of tabulations in published annual almanacs, despite the fact that it is known to be inaccurate.

Recent investigations of Phoebe's motion have been based on numerical integrations, beginning with the work of
Rose (1979) and continuing with papers by Bec-Borsenberger (1982), Bykova \& Shikhalev (1984), Jacobson (1998), and most recently, Arlot et al. (2003). The most recent papers have made use of CCD observations of Phoebe which extend the time span of the data to almost a century.

In this paper, we also rely on numerical integration rather than analytical theory in order to obtain an adequate fit to the observations. The equations of motion for the satellite are written in rectangular coordinates in a Saturnicentric reference system and are referred to the Earth mean equator and equinox of the J2000 system (ICRF). The numerical integration was calculated using the 12th-order Runge-Kutta-Nystrom formula of Brankin et al. (1989).

For Phoebe, the overwhelming perturbation is due to the Sun. In this respect, Phoebe presents a similar problem to that of the Moon's orbit around the Earth. Perturbations by the Sun, Jupiter, and Uranus were computed using their positions derived from the JPL planetary ephemeris DE406. An additional perturbation due to the oblateness (J2) of Saturn was included, although its effect is not significant at the distance of Phoebe.

Perturbations by the eight inner satellites, including Titan, were included in our numerical integration by augmenting the mass of Saturn by the masses of these satellites. We replaced the mass of Saturn alone, assumed to be $2.85815 \times 10^{-4} M_{\odot}$ (Jacobson 1998), by the mass of the whole Saturnian system, including the mass of the rings and the eight inner satellites, which is $2.85942 \times 10^{-4} M_{\odot}$ (Sinclair \& Taylor 1985).

In order to estimate the effect of Jupiter, we performed two series of integrations; the first included perturbations by Jupiter, and the second did not. 


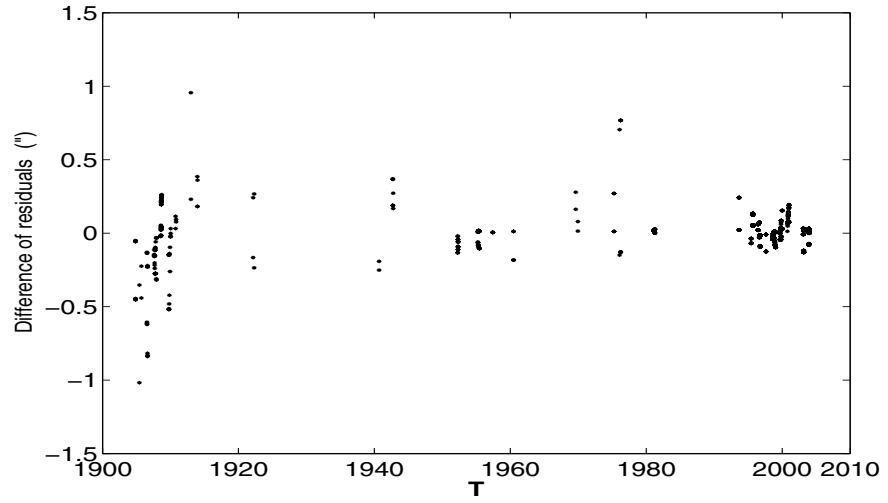

Fig. 1. The difference of $\mathrm{O}-\mathrm{C}$ residuals between an integration with Jupiter and an integration without Jupiter for the period 1904-2004.

Figure 1 shows the difference of residuals in the sense of an integration with Jupiter minus an integration without Jupiter. Although the differences in the residuals are quite noticeable in the old observations, the scatter of the residuals in those observations is also very large. The differences are smaller for the CCD observation made after 1993, but they are not significant compared to the accuracy of the observations.

However, we decided to include perturbations of Jupiter, although we have shown that they are very small. Perturbations from other planets are even smaller (Jacobson 1998) so they were not considered in this work.

\section{Fit to observations and orbit determination of Phoebe}

\subsection{Sources of the observations and weighting}

In order to best determine Phoebe's orbit, it is necessary to fit the orbital model to observations that cover the longest possible time span.

In this work, we used 686 observations covering a whole century from 1904 to 2004. Of these, 182 are photographic observations made during the period 1904-1981, taken from the Bec-Borsenberger (1982) catalogue.

The remaining data sets were obtained using CCD devices after 1981. The most recent set of observations were made by Qiao and Tang at Sheshan station in December 2003 using the $1.56 \mathrm{~m}$ reflector with a CCD chip of $2048 \times$ 2048 pixels. Another new set of observations was made by Peng et al. (2004) using the $1 \mathrm{~m}$ reflector at the Yunnan observatory in February 2003. The sources of all the observations used in this paper are listed below:

- H: 182 photographic observations made from 1905 to 1981 , and 48 CCD observations made in 1993 and 1995;

- V: 60 CCD observations made at the Laboratório Nacional de Astrofísica (LNA) in Brazil from 1995 to 1997 by Veiga $\&$ Martin et al. (2000);

- F: 162 CCD observations made at the Observatoire de Haute-Provence, France from 1998 to 2000 by Fienga et al. (2002);

- S: 84 CCD observations made at Flagstaff observatory in 1998-2001 by Stone $(2000,2001)$;

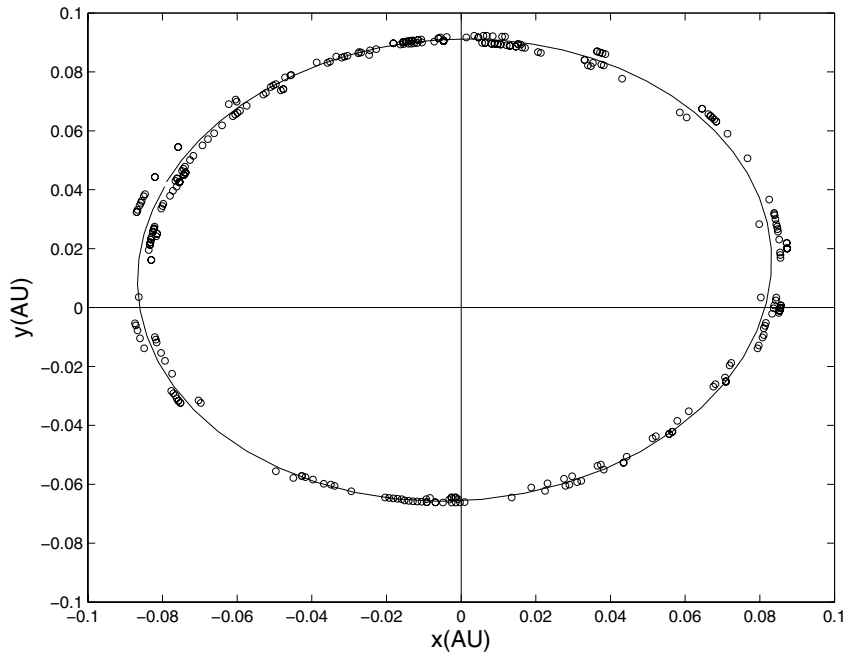

Fig. 2. Distribution for the observations on Phoebe orbit projected on the Earth's mean equator J2000.

- P: 49 CCD observations made at Yunnan observatory in February 2003 by Peng et al.(2004);

- Q: 101 CCD observations obtained at Sheshan station, SHAO in December 2003 by Qiao \& Tang (2004).

Figure 2 shows that the observations used in this paper cover the whole orbit of Phoebe. The fact that the new CCD observations are more accurate than the previous observations needs to be taken into account in the orbit fit. Thus, for a more reasonable determination of the orbit, we have assigned a different weight to each set of observations based on our assessment of the quality of the observations in the set. Assuming a unit weight for the old photographic observations, a weight of two was assigned to the later data by Fienga et al. (2002), Veiga et al. (2000) and Stone (2000, 2001), while a weight of four was assigned to the newest CCD observations sets by Peng et al. (2004) and by Qiao \& Tang (2004).

\subsection{Initial conditions and Reference frame}

The initial conditions of Phoebe were derived from the set of positions and velocities for the epoch JD 2440600.5 published by Bec-Borsenberger (1982), expressed in AU and AU/day and rotated from the B1950 reference frame to the J2000 reference frame. These are shown in Table 1.

The photographic observations were taken from the Bec-Borsenberger catalogue, which is referred to the Earth's mean equator and equinox of B1950. When using the pre1982 data, we had to rotate the vectors from J2000 to B1950 in order to compare the calculated positions directly with the observations.

The observations by Peng et al. (2004) are geocentric apparent positions at the epoch of date. Our reduction of these observations included effects such as aberration, precession, and nutation. 
Table 1. Saturnicentric state vector at JD 2440600.5 referred to the Earth mean equator and equinox of $\mathbf{J} 2000$.

\begin{tabular}{rrc}
\hline \hline & Position (AU) & Velocity (AU/day) \\
\hline$x$ & $-0.8222471500520 \mathrm{D}-01$ & $0.2850406537806 \mathrm{D}-03$ \\
$y$ & $0.3489724110733 \mathrm{D}-01$ & $0.7990650785217 \mathrm{D}-03$ \\
$z$ & $0.2605209924106 \mathrm{D}-01$ & $0.3433879021719 \mathrm{D}-03$ \\
\hline
\end{tabular}

Table 2. The resulting initial conditions of Phoebe after least square orbit adjustment for JD 2440600.5 .

\begin{tabular}{rrrc}
\hline \hline & & Position (AU) & Velocity (AU/day) \\
\hline & $x$ & $-0.8224862297361 \mathrm{D}-01$ & $0.2851041507783 \mathrm{D}-03$ \\
$\mathrm{IC} 1$ & $y$ & $0.3491378379465 \mathrm{D}-01$ & $0.7982082866034 \mathrm{D}-03$ \\
& $z$ & $0.2604054495473 \mathrm{D}-01$ & $0.3431739564262 \mathrm{D}-03$ \\
\hline & $x$ & $-0.8223548709536 \mathrm{D}-01$ & $0.2849514959246 \mathrm{D}-03$ \\
IC2 & $y$ & $0.3485714250279 \mathrm{D}-01$ & $0.7987813257737 \mathrm{D}-03$ \\
& $z$ & $0.2603226180032 \mathrm{D}-01$ & $0.3433737344265 \mathrm{D}-03$ \\
\hline & $x$ & $-0.8222905954738 \mathrm{D}-01$ & $0.2850503488894 \mathrm{D}-03$ \\
IC3 & $y$ & $0.3486032881561 \mathrm{D}-01$ & $0.7988457450202 \mathrm{D}-03$ \\
& $z$ & $0.2605021532973 \mathrm{D}-01$ & $0.3433375379172 \mathrm{D}-03$ \\
\hline
\end{tabular}

\subsection{Results of reduction, residuals and standard deviations}

In order to obtain a rapid convergence of the process of fitting the numerical integration to the observations, we adopted an iterative process. Three successive stages were performed, and Table 2 lists all three sets of initial conditions at the epoch 2440600.5 .

(i) Starting from the preliminary initial conditions at epoch 2440600.5 given in Table 1, we computed a first set of new initial conditions from the fit to the 182 old photographic observations from 1904 to 1981 . We designated this set IC1.

(ii) The second stage started from the IC1 set to compute a second set of initial positions, designated IC2, by fitting the orbit to all the observations available before 2001, including the sets H, V, F, and S defined in Sect. 3.1.

(iii) In the final stage, starting from the IC2 set, the numerical integration was fitted to all the available observations presented in Sect. 3.1. This stage included the new CCD observations made by Qiao \& Tang and by Peng et al. (2004). The resulting set of initial conditions, designated IC3, represents the new orbit of Phoebe proposed in this paper.

We fitted the integration to observations covering the time span 1904 to 2003. Figure 3 shows a plot of the O-C residuals in right ascension and declination versus time, while Table 3 lists the mean value and rms of $\mathrm{O}-\mathrm{C}$ residuals in right ascension and declination. The spread of the residuals can be as large as 2 arcsec for the old observations; and even for the most recent (1993-2003) data, it can still reach 1 arcsec.

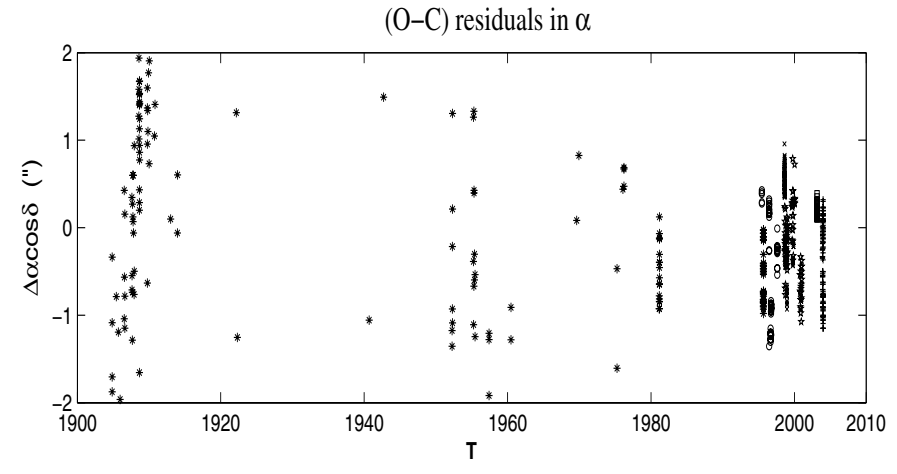

$(0-\mathrm{C})$ residuals in $\delta$

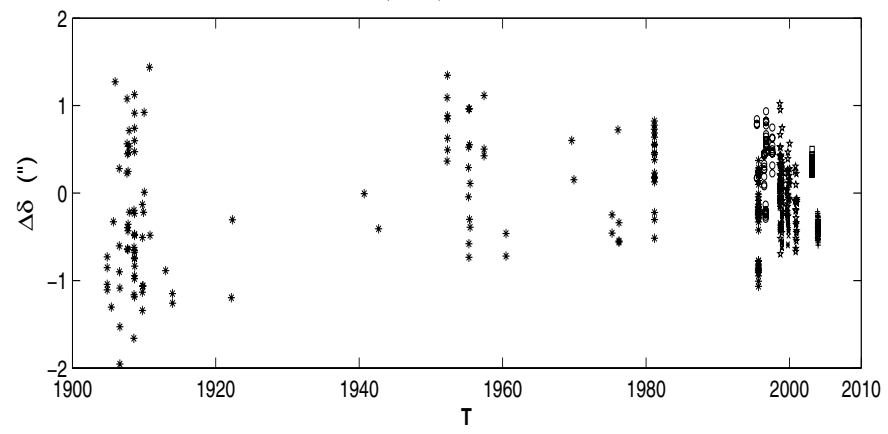

Fig. 3. The plots of $\mathrm{O}-\mathrm{C}$ residuals in right ascension and declination for the whole observations vs. $T, 1904-2004$.

Table 3. The mean residuals $\mu\left({ }^{\prime \prime}\right)$, standard deviations $\sigma\left(^{\prime \prime}\right)$ of the $\mathrm{O}-\mathrm{C}$ residuals about the mean after fitting the observations. $N_{u}$ is the number of observations used in the reduction.

\begin{tabular}{lccrr}
\hline \hline Data Set & & $N_{u}$ & $\cos \delta \Delta \alpha$ & \multicolumn{1}{c}{$\Delta \delta$} \\
\hline $\mathrm{H}(1904-1993)$ & $\sigma$ & 169 & 0.713 & 0.791 \\
& $\mu$ & 169 & -0.039 & -0.181 \\
\hline $\mathrm{V}(1995-1997)$ & $\sigma$ & 60 & 0.326 & 0.241 \\
& $\mu$ & 60 & -0.147 & 0.428 \\
\hline $\mathrm{F}(1998-1999)$ & $\sigma$ & 162 & 0.285 & 0.158 \\
& $\mu$ & 162 & 0.002 & -0.220 \\
\hline $\mathrm{S}(1998-2001)$ & $\sigma$ & 84 & 0.232 & 0.234 \\
& $\mu$ & 84 & -0.097 & 0.055 \\
\hline $\mathrm{P}(2003)$ & $\sigma$ & 49 & 0.039 & 0.050 \\
& $\mu$ & 49 & -0.026 & 0.132 \\
\hline $\mathrm{Q}(2003)$ & $\sigma$ & 101 & 0.265 & 0.063 \\
& $\mu$ & 101 & -0.054 & -0.198 \\
\hline
\end{tabular}

\section{Accuracy analysis and discussion}

\subsection{Using the UCAC2 catalogue and discussing its accuracy}

In previous satellite astrometry campaigns using CCD detectors of small size, the "brighter moon method" of calibration has been employed by many observers, including Qiao et al. (1999), Shen et al. (2001), Harper et al. (1999), and Peng et al. (2002). This method relies on positions predicted from pre-existing ephemerides. 
Qiao and Tang used a new CCD chip that provided a larger field (about $11^{\prime} \times 11^{\prime}$ ). It was installed on the $1.56 \mathrm{~m}$ reflector at the Sheshan station. As the satellite Phoebe is very distant from the primary, it is very unlikely that it will appear in the field of view with another satellite, even when using such a large CCD chip. As a result, almost all of the published positions of Phoebe are absolute coordinates, calculated by astrometric reduction from the positions of nearby catalogue stars. Consequently, the observed positions of Phoebe are very sensitive to the accuracy of the star catalogues used.

The new observations by Qiao and Tang used in this work were obtained using the wide-field CCD chip, which provides a large number of background stars. In practice, between 13 and 91 reference stars could be found on each frame, which allowed an astrometric calibration from the very accurate UCAC2 star positions.

Zacharias et al. (2004) report that the UCAC2 catalogue contains more than 48 million stars with magnitudes ranging from about 7.5 to 16 . Its nominal positional errors are about $0{ }^{\prime} 02$ for the stars in the 10th to 14th magnitude range and about $0{ }^{\prime} 07$ at the limiting magnitude of 16. Furthermore, proper motion errors are about 1-3 mas/year for the 12th magnitude, and about 4-7 mas/year for fainter stars to the 16th magnitude. No zonal error was given in the catalogue.

The new observations of Phoebe by Qiao and Tang are the first ones to be derived from such an accurate star catalogue. It is not surprising that they present a higher accuracy than most of the previous observations of Phoebe, as can be seen in Table 3, which also shows the very high quality of Peng's new observations. These two new high-quality sets of observations contribute significantly to the improvement in knowledge of Phoebe's orbit, as we discuss in the next section.

Jacobson (1998) notes that observations of Phoebe relative to other Saturnian satellites would lead to a considerable improvement in its orbit. Unfortunately, Phoebe is only in the vicinity of Saturn and its other satellites for a few days in each 550-day orbit, so such opportunities are rare. Nonetheless, we hope to make future observations of Phoebe relative to the other satellites.

\subsection{Analyzing and comparison}

In order to analyse the accuracy of our new orbit, we chose two subsets of the observations which are designated thus:

- Set A: all photographic observations of the $H$ sources.

- Set B: only the newest observation by Peng and Qiao.

The mean residuals $\mu$ and the root-mean-square of the residuals $\sigma$ were calculated, respectively, for the two sets of observations from the three successive sets of initial conditions IC1, IC2, and IC3 given in Table 2 of Sect. 3.3. The statistics are shown in Table 4. The residuals for each set of observations decrease with the use of successive sets of initial conditions IC1, IC2, and IC3. This emphasizes the efficiency of the iterative process we used for computing the numerical integration of Phoebe's orbit.

Table 4 also clearly shows that the new orbit yields very low residuals for the new observations by Qiao and Tang and
Table 4. The root mean square of the residuals $\sigma\left({ }^{\prime \prime}\right)$ and the mean error $\mu\left({ }^{\prime \prime}\right)$ for the different sets of initial conditions IC1, IC2, IC3 derived from the iterative numerical integration and for both set of observations A (old photographic) and B (recent CCD Qiao and Peng).

\begin{tabular}{ccrrrr}
\hline \hline & & \multicolumn{2}{c}{ SetA $=$ Photo } & \multicolumn{2}{c}{ SetB $=\mathrm{Q}+\mathrm{P}$} \\
& & $\alpha$ & \multicolumn{1}{c}{$\delta$} & \multicolumn{1}{c}{$\alpha$} & \multicolumn{1}{c}{$\delta$} \\
\hline IC1 & $\sigma$ & 0.694 & 0.680 & 0.790 & 0.778 \\
& $\mu$ & 0.065 & -0.167 & -0.726 & -0.412 \\
\hline IC2 & $\sigma$ & 0.717 & 0.696 & 0.282 & 0.475 \\
& $\mu$ & -0.041 & -0.082 & -0.171 & -0.224 \\
\hline \multirow{2}{*}{ IC3 } & $\sigma$ & 0.713 & 0.791 & 0.224 & 0.189 \\
& $\mu$ & -0.039 & -0.181 & -0.045 & -0.090 \\
\hline
\end{tabular}

by Peng et al. (2004). The mean error $\mu$ of these residuals are as low as a few tens of milliarcseconds (45 mas in right ascension and 90 mas in declination). These very low values demonstrate the accuracy of our new orbit at the present epoch.

Comparison of our new orbit with that of Arlot et al. (2003) shows very similar accuracy for the new observations. However, our orbit seems to have better accuracy over a longer period of time as it presents significantly lower residuals even for the old photographic observations (40 mas in right ascension and 181 mas in declination) compared to the corresponding residuals from Arlot's orbit, which are significatively higher at more than 600 mas.

\section{Conclusion}

The faintness of Phoebe (mean magnitude of 16.5 at opposition) and its great distance from Saturn mean that there are fewer than 600 observations of Phoebe in the century following its discovery. This has presented great problems to anyone who has tried to improve its orbit.

The situation has begun to change in the past ten years as many CCD observations have been made in preparation for the NASA/ESA Cassini-Huygens mission. In this paper we have re-determined Phoebe's orbit. In our reduction, we combined the old photographic observations available for the period 1904 to 1981 with the recent CCD data published by Veiga et al. (2000), Fienga et al. (2002), and Stone (2000 and 2001). In addition, we incorporated 150 new CCD observations made by Qiao and Tang and by Peng (2004) in 2003.

Fienga et al. (2002) and Arlot et al. (2003) have pointed out that the use of an accurate modern star catalogue will greatly improve the accuracy of the reduced observations. Our paper presents the first orbit of Phoebe that includes observations derived from an astrometric absolute calibration using high quality star positions taken from the new UCAC2 catalogue.

Comparison of residuals derived from our new orbit with corresponding residuals given by Arlot et al (2003) shows a rather good agreement between both of these orbits, especially at the current epoch. Furthermore, we have shown that our orbit leads to significantly lower residuals than Arlot's orbit from the 
old photographic observations. This indicates the higher accuracy of our new orbit over a long period of time.

These positive results show the significant benefit obtained by adding the new and accurate CCD observations of Qiao and Peng into our numerical integration. We wish to encourage observers to use the techniques of Qiao and Peng to make more accurate $\mathrm{CCD}$ observations in the future.

Acknowledgements. The authors wish to again thank the staff at the Sheshan station of the Shanghai Observatory for their assistance throughout our observing run. We are very grateful to Prof. E. M. Standish for his beneficial advice in our use of the JPL planetary ephemerides. Dr. Q. Y. Peng provided his very precise observations of the satellite of Saturn, and for this help the authors would like to express sincere thanks to him. The authors also wish to mention the kind response from Prof. R. A. Jacobson to our questions. This work was carried out with financial support from the National Science Foundation of China (NSFC).

\section{References}

Arlot, J.-E., Bec-Borsenberger, A., Fienga, A., et al. 2003, A\&A, 411, 309

Bec-Borsenberger, A., \& Rocher, P. 1982, A\&AS, 50, 423
Brankin, R. W., Dormand, J. R., Gladwell, I., et al. 1989, ALGORITHM 670: A Runge-Kutta-Nystrom code, ACM Trans. Math. Softw., 15, 31

Bykova, L. E., \& Shikhalev, V. V. 1984, Celest. Mech., 32, 185

Duriez, L., \& Vienne, A. 1991, A\&A, 243, 263

Elmabsout, E. 1970, A\&A, 5, 68

Fienga, A., Arlot, J.-E., Baron, N., et al. 2002, A\&A, 391, 767

Jacobson, R. A. 1998, A\&A, 128, 7

Harper, D., Beurle, K., et al. 1999, A\&A, 121, 257

Peng, Q. Y., Vienne, A., \& Shen, K. X. 2002, A\&A, 383, 296

Peng, Q. Y., Vienne, A., Han, Y. B., et al. 2004, A\&A, 424, 339

Pickering, E. C. 1899, Harv. Coll. Obs. Circ, 43

Pickering, W. H. 1905, Ann. Harv. Coll. Obs. LIII III, 45

Pickering, W. H. 1905, Ann. Harv. Coll. Obs. LIII V, 85

Qiao, R. C., Tang, Z. H., private communication

Qiao, R. C., Shen, K. X., Liu, J. R., et al. 1999, A\&AS, 137, 1

Rose, L. E. 1979, AJ, 84, 1067

Ross, F. E. 1905, Harv. Coll. Obs. LIII VI, 101

Sinclair, A. T., \& Taylor, D. B. 1985, A\&A, 147, 241

Shen, K. X., Dourneau, G., Qiao, R. C., et al. 2001, A\&A, 367, 1061

Stone, R. C. 2000, AJ, 120, 2124

Stone, R. C. 2001, AJ, 122, 2723

Veiga, C. H., Vieira Martins, R., \& Andrei, A. H. 2000, A\&AS, 142, 81

Zacharias, N., Urban, S. E., Zacharias, M. I., et al. 2004, AJ, 127, 3043

Zadunaisky, P. E. 1954, AJ, 59, 1 